\title{
Perfusión miocárdica con ecocardiografía: A la búsqueda del Santo Grial.
}

os contrastes ecocardiográficos son suspensiones de microburbujas que, inyectadas por vía intravenosa, aumentan la intensidad de la señal ecográfica mejorando sensiblemente la relación señal-ruido.

La primera descripción del "efecto de contraste" fue realizada por Gramiak and Shah en 1968, quienes observaron que las burbujas de suero salino inyectadas en aorta ascendente producían opacificación de las cavidades cardíacas ${ }^{(1)}$. A partir de estos hallazgos se investigaron los primeros agentes de contraste que eran producidos por la agitación manual de sustancias diversas como solución salina, dextrosa, peróxido de hidrógeno, etc. Sin embargo, estas sustancias sólo podían producir opacificación de las cavidades derechas, ya que eran incapaces de atravesar la barrera de la microcirculación pulmonar, donde eran destruidas.

Feisntein et al. describieron el proceso de sonicación, mediante el cual un sonido de alta energía transforma el aire dentro de un medio líquido en microburbujas ${ }^{(2)}$. Con la aplicación de esta técnica en una solución de albúmina humana fue posible obtener burbujas más estables y de un tamaño homogéneo, menor de 10 micras, lo que sentó las bases para el desarrollo del primer contraste capaz de opacificar las cavidades izquierdas.

Los agentes de contrastes de Primera Generación contenían burbujas con aire en su interior, el cual es altamente difusible y rápidamente se escapa de las mismas al mezclarse con la sangre. Esto traía como consecuencia una disminución del tamaño de la burbuja y por lo tanto una menor capacidad de dispersión del ultrasonido. Sin embargo, agentes como el Levovist, mejoraban la estabilidad de las microburbujas de aire, al incorporar una sustancia surfactante (ácido palmítico) ${ }^{(3)}$. Posteriormente se desarrollaron nuevos agentes de contraste, llamados de Segunda Generación, que contenían gases de alto peso molecular, con menos solubilidad y difusión. Estas características les conferían una mayor estabilidad y una vida media más prolongada, y son los que se utilizan en la actualidad ${ }^{(3-4)}$.

De forma simultánea al diseño de ecopotenciadores más eficaces, se produjo un importante desarrollo tecnológico en los equipos de ultrasonidos con el fin de mejorar su capacidad de detección de las microburbujas, ya que ésta era muy limitada en los equipos convencionales. Actualmente existen diversos métodos que aprovechan los fenómenos de producción de ondas armónicas por las microburbujas expuestas a los ultrasonidos para separar la señal del contraste de la señal tisular. De esta manera el uso de contraste en ecocardiografía, aunque presenta problemas de obtención del producto en algunos países latinoamericanos, se ha introducido en la rutina de muchos laboratorios a lo largo del mundo con unas indicaciones claramente definidas.

\section{El uso de ecocardiografía de contraste en la rutina del laboratorio de imagen.}

Actualmente las aplicaciones de los ecopotenciadores son muy diversas y han expandido la capacidad diagnóstica de la ecocardiografía al reducir sensiblemente los pacientes con estudios no interpretables. Se debe considerar un control de calidad de un laboratorio de ecocardiografía la utilización de ecopotenciadores para mejorar la visualización del VI al menos en un 5\% de los estudios.

En líneas generales las indicaciones admitidas de pueden resumir en estos apartados ${ }^{(5-13)}$

- Opacificación del ventrículo izquierdo y detección del borde endocardio en pacientes con mala ventana ecocardiográfico.

- Cuantificación de los volúmenes ventriculares izquierdos y fracción de eyección.

- Complicaciones mecánicas post infarto.

- Detección y caracterización de masas intracardiacas.

- Caracterización de miocardiopatías: miocardiopatía hipertrófica apical y miocardiopatía no compactada.

- Apoyo a la ablación septal en la MCH.

- Patología aórtica y específicamente la disección aortica.

- Potenciación de la señal Doppler. 


\section{A la búsqueda del Santo Grial: Perfusión miocárdica en la cardiopatía isquémica.}

El uso más provocador de las técnicas de contraste es la posibilidad de analizar la perfusión miocárdica. Es a partir de la mediados de la década de los 90 cuántos diferentes grupos de trabajo demuestran la posibilidad de obtener opacificación del miocardio izquierdo, utilizando tecnología del segundo armónico.

Tras la inyección de contraste venoso, estudios experimentales demostraban que las microcavitaciones de los diferentes agentes de contraste tenían un comportamiento extravascular muy similar al de las células rojas de la sangre ${ }^{(14)}$. Al mezclarse las microburbujas con la sangre su concentración dentro de una determinada área del miocardio izquierdo y en un determinado momento, reflejaba el volumen relativo de sangre dentro de esta región ${ }^{(15-16)}$. Aún más se podían obtener distintos datos de cuantificación dependiendo de la dinámica de entrada del flujo de burbujas en el árbol coronario: cuando se administraba un bolo de contraste intracoronario se producía un rápido aumento de intensidad de la señal en el miocardio, que ecocardiografíamente se llenaba de "ecos blancos" hasta un máximo ,después del cual la intensidad se mantenía en un tiempo y luego disminuía poco a poco, este comportamiento se define como una curva de tiempo-intensidad (17). A partir de esta curva era posible estimar el tiempo medio de tránsito del contraste en el miocardio así como el volumen de sangre del mismo. Se podía establecer una relación lineal entre la concentración de microburbujas y la intensidad de la señal generada. Los patrones de perfusión normal y anormal fueron descritos posteriormente con detalle en modelos caninos y humanos.

\section{Cuándo utilizar ecocontraste para valoración de la perfusión en cardiopatía isquémica: Los grandes "fuegos artificiales" de los estu- dios científicos}

Existe una gran cantidad de trabajos en la literatura de los últimos 20 años, que confirman la posibilidad teórica de utilización de contraste en muy diferentes situaciones en el entorno del estudio de la perfusión en la cardiopatía isquémica. De una manera muy breve los podemos agrupar en diferentes apartados:

\section{- Perfusión miocárdica en la detención la enfermedad coronaria:!!!Tan potente o superior al SPECT ii:iEl santo Grial descubierto?}

Aunque podríamos traer a esta editorial muy numerosos trabajos en la literatura, que confirman la superioridad de la técnica de ecocardiografía de contraste sobre la técnica estándar de Medicina nuclear, me voy a referir únicamente a dos poderosísimos estudios, por tratarse de estudios multicéntricos organizados por grupos muy solventes:

1-En el año 2006 un muy poderoso estudio publicado en JACC prospectivo y multicéntrico capitaneado por R. Senior ${ }^{(18)}$, nombre que esta unido a la pasión y a los grandes estudios apoyando el uso de contraste/perfusión, establece una comparación entre la ecocardiografía de contraste y el SPECT para la detección de enfermedad coronaria significativa, en pacientes con clínica sugerente de enfermedad coronaria: La conclusión de este trabajo es absolutamente clara para los autores. Se confirma que el estudio de la perfusión miocárdica con contraste ecocardiográfico es totalmente comparable a los datos de perfusión obtenidos con Medicina Nuclear en la detección de enfermedad coronaria (sensibilidad $84 \%$ versus $82 \%$, especificidad $56 \%$ versus $53 \%$ ) no solo en segmentos individuales sino también en localizar de la enfermedad por el territorio afectado y en valorar la enfermedad multivaso. Este trabajo fue realmente el primer estudio con un volumen grande de enfermos, que mostraba de una manera aparentemente definitiva que el contraste era comparable al estudio de SPECT en la definición y diagnóstico de la cardiopatía isquémica. Es importante recordar que el SPECT en USA es el primer método de diagnosticar isquemia en la rutina.

2-Por si el anterior trabajo no hubiese sido suficientemente claro, en el año 2013 se organiza un estudio europeo ${ }^{(19)}$, financiado en su totalidad por una compañía de contraste, que pretende ser el definitivo para responder a la pregunta eterna: ¿SPECT o contraste en la detección y diagnóstico de enfermedad coronaria? Se trataba de un estudio multicéntrico en el que participaban 34 centros europeos, de nuevo liderados por R. Senior, con prácticamente la totalidad de centros que habían publicado sobre Eco contraste. La conclusión del trabajo de nuevo confirmaba la sensibilidad superior de las técnicas de contraste con respecto a la medicina nuclear en la detección de enfermedad coronaria. El contraste tenía mas sensibilidad comparado con el SPECT (75,2\% versus 49,1\% p>0.0001) aunque la especificidad era inferior en lesiones $>70 \%$. Igualmente los niveles de sensibilidad para pacientes con enfermedad de un solo vaso con estenosis mayor del $70 \%$ era mejor para los estudios con contraste $72 \%$, que para la medicina nuclear 42\%: $p<0.0001$. El valor pronóstico del método aumentaba durante la dobutamina con estudio de la contracción segmentaria estudiada simultaneamente a la valoración de la perfusion.

\section{- Perfusión miocárdica en la unidad de dolor torácico y en la Unidad Coronaria.}

Dentro de las posibilidades del uso del contraste genero mucho interés la potencial utilidad en los pacientes evaluados en Urgencias por dolor torácico. El ecocontraste se podía realizar más rápidamente que otras técnicas como la detección de marcadores de daño miocárdico o una prueba de detección de isquemia. La evaluación simultánea de la perfusión miocárdica con contraste mejoraba la estratificación de 
riesgo de los pacientes con dolor torácico: los pacientes con defectos de perfusión tenían un riesgo 2.4 veces mayor de presentar eventos precoces ${ }^{(20)}$. Tanto las anomalías de contracción segmentaria como los defectos de perfusión eran predictores independientes de eventos cardiovasculares. Además, la evaluación contracción-perfusión en la unidad de dolor torácico mostraba ser coste-efectiva y así se sugería que pacientes que tenían un estudio de eco contraste normal podrían ser dados de alta directamente reduciendo la tasa de admisión en un 45\%. En la Unidad Coronaria, la valoración simultánea contracción-perfusión con ecocardiografía de contraste podría ser potencialmente muy útil. La restauración del flujo en la arteria responsable del infarto no siempre se acompaña de la normalización del flujo en la microcirculación coronaria debido a fenómenos de necrosis de los capilares o embolización distal(21). Este fenómeno, conocido como no-reflujo, es un predictor muy importante de eventos clínicos adversos y de remodelado ventricular adverso ${ }^{(22)}$. Por la distribución intravascular de los ecopotenciadores, en los estudios de perfusión miocárdica con ecocardiografía de contraste, las zonas de no-reflujo aparecen como defectos de repleción intramiocárdicos, y esta técnica parecia más precisa para predecir la recuperación miocárdica que los datos ECG y angiográficos ${ }^{(23)}$. Era atractiva la posibilidad de valorar previamente al alta en los pacientes con síndrome coronario agudo de forma simultánea dos variables con valor pronóstico: FEVI y obstrucción microvascular.

\section{- Detección de isquemia mediante el estudio de la perfusión miocárdica durante la ecocardiografía de estrés.}

En la cascada isquémica, las alteraciones de la contracción segmentaria aparecen más precozmente que la angina y los cambios ECG, pero el fenómeno más precoz que se puede detectar mediante técnicas de imagen es el defecto de perfusión. Por eso resultaba muy atractiva la posibilidad de realizar estudios de estrés con ecocardiografía de contraste combinando la detección de alteraciones reversibles de la contracción segmentaria con la de defectos inducibles de perfusión.

De nuevo la literatura confirma que la evaluación combinada contracción-perfusión con ecocardiografía aumentaba la sensibilidad del diagnóstico de cardiopatía isquémica ${ }^{(24-26)}$, mejoraba la definición de la extensión de la isquemia y por lo tanto también la predicción de eventos cardiovasculares durante el seguimiento de estos pacientes en comparación con la evaluación aislada de la contracción segmentaria.

\section{- Área en riesgo. Tamaño final del infarto.}

Tras una oclusión coronaria prolongada, la onda de necrosis progresa desde el endocardio hasta el epicardio. Si el tiempo de oclusión es breve, el infarto afecta únicamente al subendocardio y conforme la oclusión es más prolongada aumenta la transmuralidad. El tamaño final del IAM viene determinado por la duración de la oclusión coronaria y también por el tamaño del área en riesgo y la presencia de circulación colateral. La ecocardiografía de contraste podía ser el método ideal para delimitar el área funcional en riesgo durante la oclusión coronaria(27) y predecir el tamaño final del infarto(28).

A mediados de los 90, Porter ${ }^{(29)}$ fue el primero en demostrar que era posible definir el área de miocardio en riesgo mediante la administración intravenosa periférica de ecopotenciadores de segunda generación, un hallazgo confirmado posteriormente en numerosos estudios clínicos ${ }^{(30-31)}$ La ecocardiografía de contraste es por tanto muy útil para estratificar el riesgo en pacientes con IAM, diferenciando aquellos con riesgo medio-bajo, de los que presentan defectos de perfusión extensos con nula o pobre circulación colateral.

La estratificación del riesgo mediante eco contrate es mas precisa que con las variables hemodinámicas que sólo se alteran cuando el área en riesgo es importante (25-40\% del ventrículo izquierdo) o con las variables clínicas, como la fracción de eyección, que cae con un área en riesgo moderada $(18 \%)^{(32)}$.

\section{La tozuda realidad de la vida diaria: El estudio de perfusión miocárdica con contraste ecocardiográfico se hace excepcionalmen- te en la rutina:}

A pesar de los trabajos anteriores, que muestran que en la literatura existe mucha información a favor del uso del contraste como método de análisis de la perfusion miocárdica, la realidad del trabajo diario de la práctica mayoría de los laboratorios de ecocardiografía del mundo es que las técnicas de contraste para análisis de perfusión están relegadas, cuando no olvidadas en la rutina.

Se podría justificar la no utilización el eco de contraste en el ámbito latinoamericano porque en muchos países es prácticamente imposible obtener el contraste. Pero esta realidad no solo es específica de esta área sino que igualmente sucede en Europa y en los Estados Unidos.

En una reciente encuesta que realizamos en la Sociedad Española de Imagen Cardíaca y con las limitaciones que implica haberla obtenido por las redes sociales, a la pregunta ¿Utilizas el contraste ecocardiográfico para valorar la perfusión miocárdica?: El $91 \%$ de las respuestas respondían que jamás o que era una técnica absolutamente muerta, únicamente el 2\% decían que lo utilizaban de manera rutinaria y el $6 \%$ que lo hacían ocasionalmente. Creo que estos números reflejan absolutamente la realidad del método. El contraste para estudiar la perfusión miocárdica no se utiliza en la práctica de la rutina de cientos de laboratorios alrededor del mundo contradiciendo la literatura científica. Sin duda una de las máxima discrepancias entre la información de literatura científica y su aplicación en la rutina diaria, es la utilización del contraste para valorar la perfusion miocárdica. Existe un sin fin de razones que pueden explicar en parte estas discrepancias, que voy a tratar de enumerar: 
1. La técnica de contraste para perfusión miocárdica es muy manual dependiente: exige ser extraordinariamente cuidadoso desde la preparación del producto y con los diferentes métodos de administración del material. La vía de administración en bolo o por infusión continua. La colocación de los distintos setting del aparato para evitar atenuaciones o bajos niveles de señal. La frecuencia de emisión del impulso ultrasónico en tiempo real o con diferentes triggering, la duración de los pulsos de la señal. El análisis visual o cuantitativo de los resultados.

2. Existe un caos tecnológico que se ha mantenido a lo largo de los años, cada compañía ha tratado de ofrecer sus sistemas tecnológicos para realizar los estudios de contraste: power modulation, tissue acoustic emisión, inversión de fase, power Doppler, imagen coherente, inversión de pulsos. Toda esta oferta ha producido una falta de homogeneidad de los estudios, que no ha facilitado su difusión.

3. La pasión poco critica de los estudios cientíicos que parecen situar como lo normal lo que es excepcional. Nuestro grupo trabajo durante años en el estudio de la perfusion miocárdica con contraste, incluso publicamos un texto de gran difusión en Ingles sobre el método (33). Tenemos por tanto una amplia experiencia en el estudio de la perfusion del miocardio. Es cierto que muchas veces los estudio cuantitativos exigían una dedicación y esfuerzo notable, con horas en el análisis de lo estudios, becarios y personal especifico dedicado: los resultados eran óptimos en la producción científica pero olvidábamos que no eran trasladables por su complejidad a la "vida diaria".

Un ejemplo, que puede ayudar a comprender la escisión entre ciencia y rutina: Nuestro grupo describió por primera vez que el contraste post angioplastia primaria era el mejor predictor de la recuperación funcional o de viabilidad miocardica(23). Cada estudio lo realizaba una persona rigurosamente entrenada para realizar el contraste tras abrir la arteria responsable. El análisis de las curvas de perfusion Ilevaba literalmente horas de estudio. Sin duda el resultado era científicamente cierto pero de nula aplicación clínica.

Los estudios de contraste/perfusión se mantiene ahora en un muy limitado mundo formado por escasos grupos, básicamente de Italia, Inglaterra y USA, con gran poder de presión, que mantiene una llama iniciática, una antorcha que cada vez esta mas alejada del mundo real, al menos para el resto del mundo.

\section{¿Existe futuro para el contraste en ecocardiografía?}

Debe haber un cambio importante en el uso de las técnicas de contraste para que estas puedan utilizarse de una manera rutinaria en la valoración de la perfusión miocárdica. En mi opinión solo hay dos posibilidades para que se produzca este cambio: o bien una mejoría de los contrastes o bien una mejoría tecnológica.

Con respecto a la primera personalmente creo que no se necesita una mejoría en la calidad de las microcavitaciones. Efectivamente los contrastes actuales producen importantes ecos de backscatter generados por las vibraciones de las burbujas, que son los que detectamos con el ecocardiograma. No necesitamos que las burbujas resuenen más, sino que tecnológicamente seamos capaces de detectar cambios mínimos de la señal generada por la vibración de las microcavitaciones. La incorporación de la inteligencia artificial y la radiomica en el estudio de las modificaciones que se produce en el miocardio tras el paso de contrate probablemente tenga la solución.

El estudio con Inteligencia Artificial de los cambios en la textura, tramas, intensidad, estructura y decenas de parámetros, obtenidos y comparados antes y después del contraste, en los diferentes segmentos del miocardio, podrían ser teóricamente el camino que nos lleve al Santo Grial, aunque nadie todavía se ha lanzado por este via. El uso de la Radiomica, que no es otro que ir mas allá de lo que ve el ojo humano, está dando unos resultados espectaculares en otros campos de la imagen y podría igualmente ser aplicado en el estudio de la perfusión miocárdica con ecocardiografía.

De estos cambios dependerá que el contraste para el estudio de la perfusion miocardica tenga esperanza de vida o sea simplemente, como lo es ahora, un fuego artificial sin impacto en la rutina de los estudios de nuestros enfermos. Solo el tiempo nos dará la respuesta.

Prof. MA García Fernández 


\section{Prof. MA García Fernández}

Catedrático de Imagen Cardiaca Departamento de Medicina

Universidad Complutense

Madrid

España
Correspondencia

M.A. García Fernández

maecocardio@gmail.com

Citar como: García Fernández MA. Perfusión miocárdica con ecocardiografía: A la búsqueda del Santo Grial. RETIC. 2021 (Diciembre); 4 (3): I-VI. doi: 10.37615/retic.v4n3a1

Cite this as: García Fernández MA. Myocardial perfusion with echocardiography: In search of the Holy Grail. RETIC. 2021 (Diciembre); 4 (3): I-VI. doi: 10.37615/retic.v4n3a1

\section{Referencias}

1. Gramiak R, Shah PM. Echocardiography of the aortic root. Invest Radiol. 1968 Sep-Oct;3(5):356-66.

2. Feinstein SB, Ten Cate FJ, ZwehI W, Ong K, Maurer G, Tei C, et al. Two-dimensional contrast echocardiography. I. In vitro development and quantitative analysis of echo contrast agents. J Am Coll Cardiol. 1984 Jan;3(1):14-20.

3. Serra V, García Fernández M, Zamorano J. Microbubbles: basic principles. Contrast echocardiography in Clinical Practice Springer Verlag; 2004 . p. 19-44.

4. Cheng SC, Dy TC, Feinstein SB. Contrast echocardiography: review and future directions. Am J Cardiol. 1998 Jun 18;81(12A):41G-8G.

5. Garcia-Fernandez MA, Macchioli RO, Moreno PM, Yanguela MM, Thomas JB, Sendon JL, et al. Use of contrast echocardiography in the diagnosis of subacute myocardial rupture after myocardial infarction. J Am Soc Echocardiogr. 2001 Sep;14(9):945-7.

6. Waggoner AD, Williams GA, Gaffron D, Schwarze M. Potential utility of left heart contrast agents in diagnosis of myocardial rupture by 2-dimensional echocardiography. J Am Soc Echocardiogr. 1999 Apr;12(4):272-4.

7. Nakatani S, Imanishi T, Terasawa A, Beppu S, Nagata S, Miyatake K. Clinical application of transpulmonary contrast-enhanced Doppler technique in the assessment of severity of aortic stenosis. J Am Coll Cardiol. 1992 Oct;20(4):973-8.

8. Ha JW, Lee BK, Kim HJ, Pyun WB, Byun KH, Rim SJ, et al. Assessment of left atrial appendage filling pattern by using intravenous administration of microbubbles: comparison between mitral stenosis and mitral regurgitation. J Am Soc Echocardiogr. 2001 Nov;14(11):1100-6.

9. Fernandez Portales J, Garcia Fernandez MA, Moreno M, Gonzalez Alujas MT, Placer JL, Allue C, et al. [Usefulness of the new imaging techniques, second harmonic and contrast in endocardial border visualization. Reliability analysis in segmental contraction assessment]. Rev Esp Cardiol. 2000 Nov;53(11):1459-66.

10. Reilly JP, Tunick PA, Timmermans RJ, Stein B, Rosenzweig BP, Kronzon I. Contrast echocardiography clarifies uninterpretable wall motion in intensive care unit patients. J Am Coll Cardiol. 2000 Feb;35(2):485-90.

11. Yong Y, Wu D, Fernandes V, Kopelen HA, Shimoni S, Nagueh SF, et al. Diagnostic accuracy and cost-effectiveness of contrast echocardiography on evaluation of cardiac function in technically very difficult patients in the intensive care unit. Am J Cardiol. 2002 Mar 15;89(6):711-8.

12. Kusnetzky LL, Khalid A, Khumri TM, Moe TG, Jones PG, Main ML. Acute mortality in hospitalized patients undergoing echocardiography with and without an ultrasound contrast agent: results in 18,671 consecutive studies. J Am Coll Cardiol. 2008 Apr 29;51(17):1704-6.

13. Thomson HL, Basmadjian AJ, Rainbird AJ, Razavi M, Avierinos JF, Pellikka PA, et al. Contrast echocardiography improves the accuracy and reproducibility of left ventricular remodeling measurements: a prospective, randomly assigned, blinded study. J Am Coll Cardiol. 2001 Sep;38(3):867-75.

14. Lepper W, Hoffmann R, Kamp O, Franke A, Cock CC, Kühl HP, Sieswerda GT, Dahl JV, Janssens U, Voci PVisser CA, Hanrath P. Assessment of myocardial reperfusion by intravenous myocardial contrast echocardiography and coronary flow reserve after primary percutaneous transluminal coronary angiography in patients with acute myocardial infarction. Circulation 2000; 101: 2368-2374.

15. Jayaweera AR, Edwards N, Glasheen WP, Villanueva FS, Abbott RD, Kaul S. In vivo myocardial kinetics of air-filled albumin microbubbles during myocardial contrast echocardiography. Comparison with radiolabeled red blood cells. Circulation Research 1994; 74: 1157-1165

16. Jayaweera AR, Matthew TL, Sklenar J, Spotnitz WD, Watson DD, Kaul S. Method for the quantitation of myocardial perfusion during myocardial contrast two-dimensional echocardiography. J Am Soc Echocardiogr 1990;3(2):91-98.

17. Cheirif J, Zoghbi WA, Raizner AE, Minor ST, Winters WL Jr, Klein MS, De Bauche TL, Lewis JM, Roberts R, Quiñones MA. Assessment of myocardial perfusion in humans by contrast echocardiography. I. Evaluation of regional coronary reserve by peak contrast intensity. J Am Coll Cardiol. 1988;11(4): 735-743.

18. Amarjit J , Michael Hickman, O Kamp, R M. Lang, J D. Thomas, M A. Vannan, Je Vanoverschelde, Poll A. Van Der Wouw, Roxy Senior*Myocardial contrast echocardiography for the detection of coronary artery stenosis - A prospective multicenter study in comparison with single-photon emission computed tomographyJournal of the American College of Cardiology 47(1):141-5

19. Senior R. Moreo A. Gaibazzi N. Agati L. Tiemann K. Shivalkar B. et al. Comparison of sulfur hexafluoride microbubble (SonoVue)-enhanced myocardial echocardiography to gated single photon emission computerized tomography for the detection of significant coronary artery disease: a large European multicentre study. J Am Coll Cardiol. 2013; 62: 1353-1361 
20. Rinkevich D, Kaul S, Wang XQ, Tong KL, Belcik T, Kalvaitis S, et al. Regional left ventricular perfusion and function in patients presenting to the emergency department with chest pain and no ST-segment elevation. Eur Heart J. 2005 Aug;26(16):1606-11.

21. Rezkalla SH, Kloner RA. No-reflow phenomenon. Circulation. 2002 Feb 5;105(5):656-62.

22. Perez David E, Garcia Fernandez MA. Myocardial contrast echocardiography in acute myocardial infarction: the importance of assessing coronary microcirculation. Rev Esp Cardiol. 2004 Jan;57(1):4-6.

23. Perez-David E, Garcia-Fernandez MA, Quiles J, Mahia P, Lopez-Sendon JL, Lopez de Sa E, et al. Usefulness of quantitative myocardial contrast echocardiography for prediction of ventricular function recovery after myocardial infarction treated with primary angioplasty. Heart. 2006 May;92(5):693-4.

24. Tardif JC, Dore A, Chan KL, Fagan S, Honos G, Marcotte F, et al. Economic impact of contrast stress echocardiography on the diagnosis and initial treatment of patients with suspected coronary artery disease. J Am Soc Echocardiogr. 2002 Nov;15(11):1335-45.

25. Moir S, Haluska BA, Jenkins C, Fathi R, Marwick TH. Incremental benefit of my cardial contrast to combined dipyridamole-exercise stress echocardiography for the assessment of coronary artery disease. Circulation. 2004 Aug 31;110(9):1108-13.

26. Elhendy A, O'Leary EL, Xie F, McGrain AC, Anderson JR, Porter TR. Comparative accuracy of real-time myocardial contrast perfusion imaging and wall motion analysis during dobutamine stress echocardiography for the diagnosis of coronary artery disease. J Am Coll Cardiol. 2004 Dec 7;44(11):2185-91.

27. Masugata H, Peters B, Lafitte S, Strachan M, Ohmori K, DeMaria AN. Quantitative assessment of myocardial perfusion during graded coronary stenosis by real-time myocardial contrast echo refilling curves. J Am Coll Cardiol 2001; 37: 262-269.

28. Lafitte S, Higashiyama A, Masugata H, Peters B, Strachan M, Kwan OL, DeMaria AN. Contrast echocardiography can assess risk area and infarct size during coronary occlusion and reperfusion: experimental validation. J Am Coll Cardiol 2002; 39: 1546-1554.

29. Porter TR, Xie F, Kricsfeld A, Kilzer K. Noninvasive identification of acute myocardial ischemia and reperfusion with contrast ultrasound using intravenous perfluoropropane-exposed sonicated dextrose albumin. J Am Coll Cardiol 1995;26(1):33-40.

30. Meza M, Greener Y, Hunt R, Perry B, Revall S, Barbee W, et al. Myocardial contrast echocardiography: reliable, safe, and efficacious myocardial perfusion assessment after intravenous injections of a new echocardiographic contrast agent. Am Heart J 1996;132(4):871-881.

31. Coggins MP, Sklenar J, Le DE, Wei K, Lindner JR, Kaul S. Noninvasive prediction of ultimate infarct size at the time of acute coronary occlusion based on the extent and magnitude of collateral-derived myocardial blood flow. Circulation 2001;104(20):2471-2477.

32. Kaul S, Glasheen W, Ruddy TD, Pandian NG, Weyman AE, Okada RD. The importance of defining left ventricular area at risk in vivo during acute myocardial infarction: an experimental evaluation with myocardial contrast two-dimensional echocardiography. Circulation. 1987;75(6):1249-1260,

33. GarciaFernandez MA, Zamorano J.Contrat Echocardiography in clinical Practice.Spring Verlag Berlin 2004. 